\title{
Absolute Calibration of In Vivo Measurement Systems
}

\author{
D.A. Kruchten \\ D.P. Hickman
}

February 1991

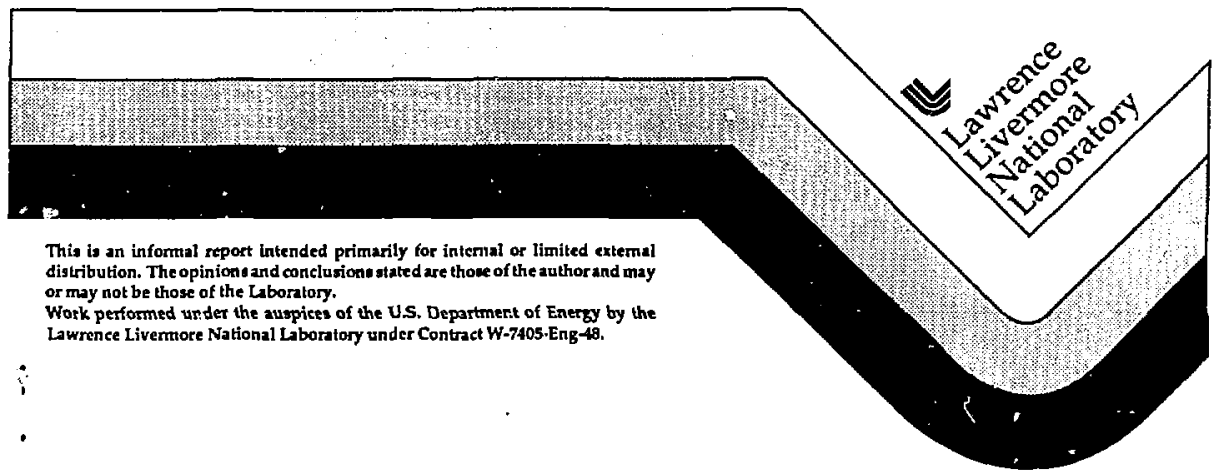


Tive socument was prepared as an acsount of work sponsored by an agency of the United States Covernment. n, sither the United Stales Government nor the University of Californta nor any of their employees, makes any warranty, express of implied, or sssumes any legal liability or cesponsibility for the dccuracy, completentss, of usefulness of any information. apparatug, producl, or prosess disclosed, or represenis that its use would not infringe prtyately owned rights. Reference herein to any specific commerciat products, process, or service by trade name, trademark, manutaclurer, or otherwise, does not necessarily constitute or imply its endorsement, recommendation, or favoring by the United States Government or the University of California. The views and opinions of authors expressed herein do not necessarily state or reflect those of the United States Covernment or the Universily of Californis, and shall not be used for advertising or product endorsement purposes.

This report has been reproduced

direslly fram the best arailable copy.

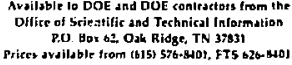

Avaluble lo ite publiti frm the

Natianal Technical Inforaulion Secrice

US. Departmenl of Commeree

5295 Pott Royjl Rd.

Springfiedd is 221 bl

Price

Code

A01

\section{Papercopy Prices}

A02

A03

A04

Aus

A06

A07

A08

A09

A 10

A11

A12

A13

A14

Al5

A:6

A17

A18

A19

A20

A21

A22

A23

A2:

A25

A99
Page

Range

Microfiche 


\section{Absolute Calibration of In Vivo Measurement Systems}

\section{Abstract}

Lawrence Livermore National Laboratory (LLNL) is currently investigating a new method for obtaining absolute calibration factors for radiation measurement systems used to measure internally deposited radionuclides in vivo. Absolute calibration of in vivo measurement systems will eliminate the need to generate a series of human surrogate structures (i.e., phantoms) for calibrating in vivo measurement systems. The wissolute calibration of in vivo measurement systems utilizes magnetic resonance imaging (MRI) to define physiological structure, size, and composition. The MRI image provides a digitized representation of the physiological structure, which allows for any mathematical distribution of radionuclides within the body. Using Monte Carlo transport codes, the emission spectrum from the body is predicted. The in vivo measurement equipment is calibrated using the Monte Carlo code and adjusting fo: the intrinsic properties of the detection system. The calibration factors are verified using measurements of existing phantoms and previously obtained measurements of human volunteers.

\section{Introduction}

Medical imaging of the human body has been greatly enhanced with the development of MRI. The three-dimensional information obtained using MRl provides an exact measure of physical size, shape, and composition of human organs and tissues. These images are in digital format, which allows computer enhancement and interpretation. MRl is a relatively new medical imaging modality, and its use has significantly increased over the past few years in the United States. An advantage of MRI over other similar imaging modalities (e.g., computerized tomography [CT]) is that it provides increased detail of physiological structure with no known physical risk to the patient.

With the increased use of MRI comes a data base of information regarding physiological structure and composition. This data base of digitized images is an ideal data pool for develepment of a mathematical set of exacting human surcogate structures (i.c., phantoms). This set of phantoms could then be used to define a "stand ard" phantom for use by the nuclear industry; to generate in vivo measurement calibration factors for internally deposited radionuclides.

The digitized images obtained from MRI are used mathematically to define source locations and distributions according to the physiology and biokinetics of the calibration radionuclide, thus providing a digitized geonetry to be used by a continuous-energy Monte Carlo transport code. The Monte Carlo code would apply corrections for attenuation through various physiological structures and tissue compositions as determined from the MRl image data. This results in a photon emission spectrum out of the body that would be available for detection by in vion measurement systems.

When a continuous-encrgy Monte Carlo code is used in conjunction with response characteristics of the detector system, the absolute efficiency for the deector system is determined for the MR! image (i.e., standard phantoms). The continuous-energy code allows for correction of the photon emission spectrum for photepeak broadening (i.e., resolution) and provides the absolute efficiency for any region of interest

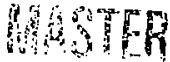


chosen within the photopeak area. Daughter radionuclides and other interfering radionuclides can also be incorporated into the source code, thereby providing an absolute calibration for any combined spectrum of radionuclides.

Verification of the absolute calibration technique for in vivo measurement systems is necessary before routine use at nuclear facilities. Currently, there are several human surrogate structures (i.e., constructed phantoms) and human volunteer radionuclide studies that can be used for verification. MRI scans are planned using the thoracic phantom developed by LLNL and the U.S. transuranium registry case 102 bone phantom.

Using these scans and known information regarding the radionuclide distribution within the various organs of these phantoms, a source geometry will be established for use by the Monte Carlo code in determining absolute calibration values. These absolute calibration values will be compared to measured calibration values for these phantoms.
In the 1980 s, in vivo measurement studies using human volunteers who inhaled $92 \mathrm{~m} \mathrm{Nb}$ were compared to each other. MRI scans were performed to define physiological and compositional structures of the volunteers. As part of the verification process for the absolute calibration technique, the intercomparison data will be reevaluated relative to computed calibration values.

In summary, the absolute calibration technique currently under investigation eliminates the need to physically construct human surrogates and can be used to define a standardized phantom for routine use by in vizo measurement systems. For nonroutine use (i.e., a measured intake of radioactive material), an MRI scan obtained for the individual containing the radioactive material could be used to determine exact calibration parameters according to the individual's physiological structure and composition.

\section{Magnetic Resonance Imaging}

\section{Concept}

Nuclear magnetic resonance (NMR), the predecessor to MRI, is a powerful tool used to identify the structure of chemicals, as well as interactions between nuclei of materials and biological systems. Although conventional applications of NMR do not allow the location of magnetically stimulated nuclei to be determined, recent developments have made imaging possible by spatially encoding the emitted signal from specific nuclei in the sample.

MRI, as this process became known, uses the signals emitted from resonating nuclei (most commonly hydrogen) in the body to create an image of all or part of the tody. Spatial information is obtained by applying gradient (or varying) magnetic fields over a stronger, uniform magnetic field. The gradient fields cause the nuclei at each position in the body to resonate at different frequencies. 'The signal receiver is tuned to accept a specific frequency; thus, when signals are received, they are a direct indication of the position from which they were generated. Signal relaxation times depend on the molecular environment of the tissue and, hence, the type of tissue from which the signal was generated.

The mid-twentir ch century brought about the first successful NMR experiments in solids and liquids. Rapidly improved methods were developed, and soon the idea of controlling the nonuniformity of magnetic fields to identify the spatial coordinates of nuclei spawned the generation of three-dimensional images. Soon the medical community began to leam of the usefulness of this technique, and in 1973, the first MRI images wrre published. 1

\section{Current State-of-the-Art}

Today, MRI surpasses other imaging techniques. MRI is more versatile and retains all of the positive aspects of other imagining techniques without retaining the known disadvantages. The spatial resolution is improving daily and is comparable to, if not surpassing, $C T$ imaging. The contrast of MRI exceeds 500 percent in soft tissue, thus providing excellent anatomical detail. ${ }^{2}$ This anatomical detail is 
the predominate feature of MRI that is used to establish a realistic phantom for Monte Carlo computations of photon transmissions in the body.

\section{Development of a Mathematical Phantom}

The digitized, three-dimensional spatial representation of the human subject is the basis for constructing a mathematical phantom to be used by a Monte Carlo code. Standard imaging techniques will (1) be used to define organ/tissue structure and interfaces in three-dimensional space, and (2) supply exact tissue density data for determining photon capture, scatter, and leakage probabilities.

The digitized information obtained from MRI, known as voxels, provide exact data on the material characteristics encountered by the histories of photons as they are guided by Monte Carlo probability. The MRI voxels transmitted from medical procedure tapes also provide shell images that can assist in visualization and optimization of the calibration process. The voxel information and images are retained and stored on a VAX 3200 computer system.

\section{Photon Emission Computations}

\section{Concepts}

Monte Cario techniques have been extensively used to describe radiation transport in matter. Most Monte Carlo techniques use the macroscopic principles and formulations to establish a probability distribution of interaction for a large number of photons. This distribution provides boundary conditions for interactions. The interaction of a single photon within this probability distribution is determined using a random number generator, which is analogous to throwing dice. Interactions are defined according to the type (i.e., scatter, absorption, or leakage). Histories of each photon are maintained, and as more histories are ottained, photon distributions become better known. The quantities of interest (c.g. photon flux and energy flux) are tallied along with estimates of statistical precision for the results.

\section{Current State-of-the-Art}

Monte Carlo codes are used to calibrate radiation detection systems. Some facilities use Monte Carlo techniques exclusively to calibrate measurement systems and use the National
Institute of Standards and Technology (NIST) traceable sources to check the calibration. With improved microprocessors, the computational codes are faster and continue to be programmed onto smaller and smaller computer systems. The operation of Monte Carlo codes on a VAX 3200 workstation is an example of new technology interfacing with improved progra zuming concepts. Libraries of nuclear data reactions and branching fractions for radioactive daughters have been developed, thereby enhancing repetitive operations and allowing computations of photon fluxes for mixtures of radionuclides.

\section{Interfacing with a Mathematical Phantom}

Monte Carlo code requires source specification. The degree of accuracy in the fina! result is highly dependent on the source specification, geometry, and reaction probabilities as photons traverse tissue. Voxels of data ane used to describe the three-dimessional location and composition o( sources and attenuating tissues/ organ. As photons tra verse a voxel, its reaction probability is controlled by the physical attenuation parameters. 


\section{Determination of Intrinsic Detector Parameters}

\begin{abstract}
Detector response functions that are calculated from first principles using Monte Carlo techniques do not account for inefficiencies in the detcetor system (i.e., light collection efficiency of the $\mathrm{NaI}$ detector); however, many codes allow input of a correction factor to account for inefficiencies. Alternatively, the response function of the detector car be measured and folded into the Monte-Carlo-computed incident photon flux spectrum. ${ }^{3-5}$ Each of the two techniques has advantages and disadvantages. For instance, folding the measured response function into the computed incident photon spectrum does not correct for scatter from nearby structures. Both of these techniques are being investigated to evaluate the optimum method for
\end{abstract}

\author{
generating efficiency factors for in vivo \\ measurement systems. \\ LI.NL has $\mathrm{HpGe}$, Phoswich, and large crystal \\ $\mathrm{Na}$ detection systems. The incident photon \\ spectrum computed using Monte Carlo codes is \\ combined with measured responses and/or \\ computed detector response functions to gener- \\ ate individual detector efficiency factors. The \\ response function is then used to assess indi- \\ vidual detector efficiency factors. The computed \\ efficiency factors will be compared to measured \\ efficiency factors for the various types of calibra- \\ tion phantoms used at LLNL (see the following \\ section). From these comparisons, the optimum \\ method for calibration of in vivo measurement \\ systems can be selected.
}

\section{Verification of Absolute Calibration Technique}

Verification of the absolute calibration technique has been considered. Currently several types of in vivo measurement phantoms exist. These phantoms could be biomedically imaged/ digitized and used as mathematical source terms for the Monte Carlo photon emission computations. The efficiency will be determined for the computed photon emissions spectrum and compared to the measured spectrum of each phantom using the various detector systems available at LLNL.

Further verification may be possible using data collected for human volunteers who inhaled

\begin{abstract}
$52 \mathrm{~m} N \mathrm{Nb}$. MRI scans of these volunteers were obtained prior to inhalation of $92 \mathrm{~m} \mathrm{Nb}$. These images could be used to establish each individual's mathematical source term for the Monte Carlo computations. Efficiency factors could then be computed for the LLNL detectors. Because LLNL participated in intercomparison tuleasurcments of these volunteers, the measured efficiency factors could then be compared to the computed efficiency factors. This verification would provid strong evidence for the validity of the absolute calibration technique.
\end{abstract}

\section{Discussion and Conclusions}

Biomedical imaging techniques are capable of defining detailed structures for the in vivo measurements. A magnetic resonance image could be produced for each in vivo measurement subject and an exact ph siltom representation produced for that individual. Alternatively, an "average" phantom could te established for routine in vizo measurements (i.e., measurements that demonstrate no detectable activity), thus eliminating the need to generate an MRI scan of each individual. An average phantom will be obtained by evaluating the data base of MRI scans cumently available.

Monte Carlo techniques have previously been used to compute absolute measurement efficiencies for in vivo measurement systems. . $^{6-8}$ However, these previous computations used theoretical phantoms that were physiologically inadequate (e.g., both lungs were of equal size). Biomedical imaging will provide an exact replication of the individual physiology. Organ sizes, shapes, and tissue compositions (i.e., fat, muscle, 
and bone) will be realistic for the individual being measured. Assumptions for establishing absolule calibration factors will be limited to considerations of radionuclide distribution between and within organs/tissues.

NIST has proposed to standardize in vivo measurements using a selected number of phantoms. The absolute calibration technique will extend beyond the concept of constructing singular phantoms to simulate actual human structure. The absolute calibration will be more exacting by using the measured human structure. In fact, this technique could serve as the NIST standard.

The final development stage for this research project will be to apply the absolute calibration technique to in vivo measurement system computer operations. This would allow absolute calibrations using simple computing equipment such as personal computers. This final development stage will provide users with a simple environment for the calibration of site-specific in vizo measurement equipment. An Autocad computer program can also be used to visualize the conceptual phantom and radionuclide source distributions relative to detector placement.

Absolute calibration of in vivo measurement systems is the final end point for developing photon transmission phantoms, which can be "average" phantoms or individually specific phantoms with exacting detail. Construction of phantoms will be computational rather than physical, making phanlom construction economjcally feasible. Radioactive source distributions can be placed into any organ/tissue or any group of organs. Source terms can be treated either as macro distributions or micto distributions. The absolute calibration technique allows for calibration of different types and configurations of detectors, including future designs of in vivo measurement systems.

\section{References}

1. P. C. Lautterbur, "Image Formation by Induced Local Interactions: Examples Employing Nuclear Magnetic Resonance," Nature 242, 190 (1973).

2. A. R. Margulis, C. B. Higgins, L. Kaufman, and L. E. Crooks (Eds.), Clinical Magnetic Resonance Imaging (Radiation Research and Education Foundation, San Francisco, CA, 1983).

3. L. Koblinger, "Monte Carlo Calculations of Scattered X-Rays From Snyder Phantoms," Health Phys. 28(6), 751-754 (1975).

4. S. Bhati, R. C. Sharma, and S. Somasundaram, "Monte Carlo Calculations of the Response uf an External Detector to a Photon Source in the Lungs of a Heterogeneous Phantom," Health Phys. 37(1). 145-159 (1979).

5. R. E. Goans and G. G. Warner, "Monte-Carlo Simulation of Photon Transport in a Heterogeneous Phantem; Application to Chest Counting of Pu And Am," Health Phys. 37(4), 533-542 (1979).

6. G. P. Estes, R. G. Schrandt, and J. T. Kriesc, Automated MCNP Photon Generation for Arbitrary Confiyzrations of Radioactive Materials and First-Principles Calculations of Photon Detector Responses, LA-11153MS/UC-34c (1988); available from NTIS, 5285 Port Royal Road, Springfield, VA 22161.

7. M. V. Green, R. L. Aamodt, and G. S. Johnston, "Monte Carlo Calculation of the Absolute Maximum Detection Efficiency of a Cylindrical Gamma Radiation Detcctor," Health Phys. 28(5), 624-628 (1975).

8. C. S. Chen, K. Doi, C. Vyborny, H. P. Chan, and G. Holje, "Monte Carlo Simulation Studios of Detectors Used in the Measurement of Diagnostic X-ray Spectra, Med. Phys. 7(6),627-635 (1980). 


\section{Bibliography}

Briesmeister, J. F., Ed. (1986), MCNP-A General Monte Carlo Code for Neutron and Photon Transport, Los Alamos National Laboratory, Los Alamos, NM, LA-7396-M/UC-34; available from NTIS, 5285 Port Royal Road, Springfield, VA 22161.

Nelson, W. R. (1986), "EGS4-A Monte Carlo Code to Simulate Electron-Photon Transport," Health Phys. 51(2).

Pandcy, L. N., and M. L. Rustgi (1987), “Long S.A.T. Monte Carlo Study of Electron Spectra Produced in Semi-lnfinite and Finite Water Phantoms Irradiated by Photons of Energies up to 2 Mev," Health Phys. 53(2), 163-174.

Humphrcys, E. R., and J. L. Humm (19838), "A Monte Carlo A pproach to the Microdosimetry of Ra-224 in Murine Compact and Cancellous Bone," Health Phys. 54(6), 607-615.

Chan, H. P. and K. Doi (1984), "Raoiation Dose in Diagnostic Radiology: Monte Carlo Simulation Studies," Med. Phys. 11(4), 480-490.

Chan, H. P., and K. Doi, (1985), "Thysical Characteristics of Scattered Radiation in Diagnostic Radiclogy: Monte Carlo Simulation Studies," Med. Phys. 12(2), 152-165.

Chan, H. P., Y. Higashida, and Y.. Doi (1985), "Performance of Antiscatter Grids in Diagnostic Radiology: Experimental Measu rements and Monte Carlo Simulation Studies," Med. Phys. 12:47, 449-454. 